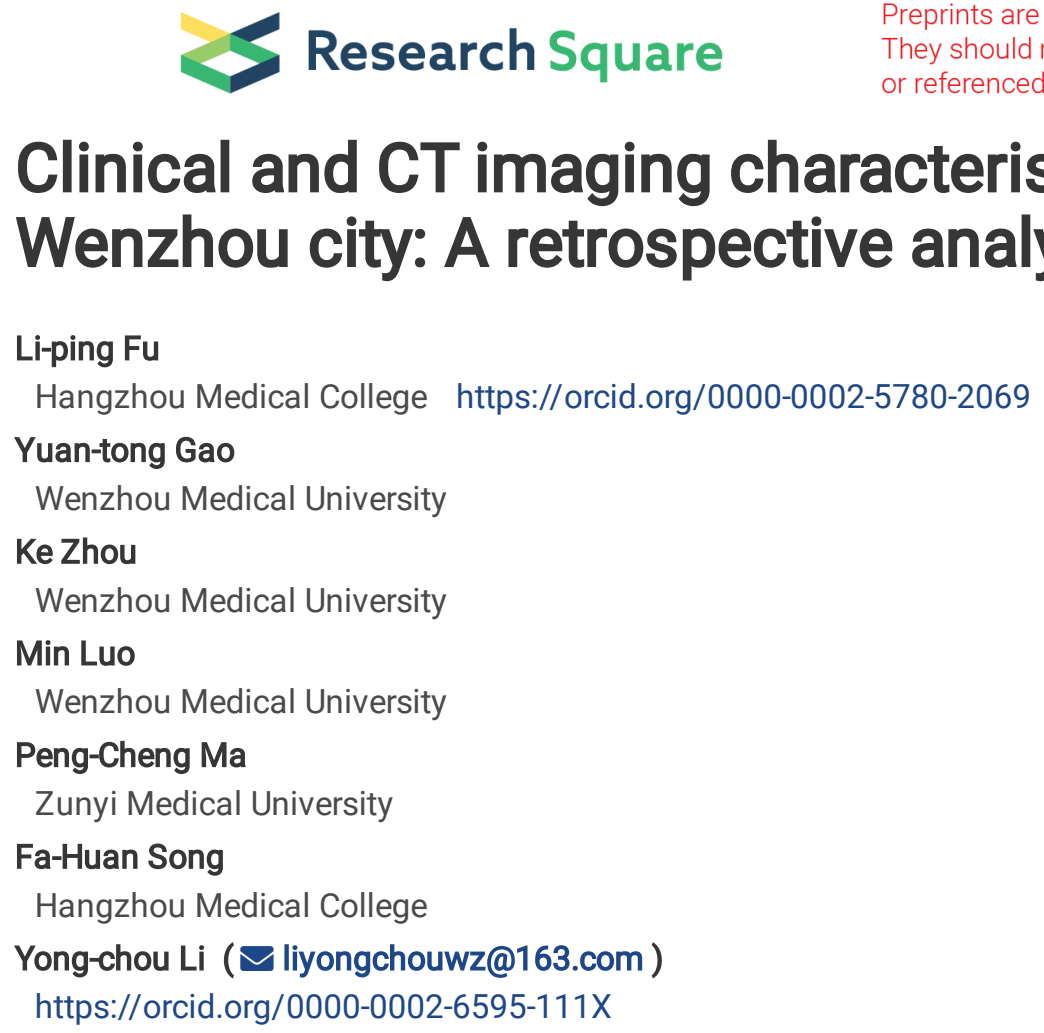

\title{
Clinical and CT imaging characteristics of COVID-19 cases in Wenzhou city: A retrospective analysis
}

Research article

Keywords: Human-human transmission, COVID-19, Clinical characteristics, Computed Tomography, Treatment

Posted Date: March 24th, 2020

DOI: https://doi.org/10.21203/rs.3.rs-18696/v1

License: @( (1) This work is licensed under a Creative Commons Attribution 4.0 International License. Read Full License 


\section{Abstract}

Background: In December 2019, Wuhan witnessed the outbreak of an "unexplained pneumonia" caused by a novel coronavirus strain infection and was dubbed the COVID-19 by the WHO. The disease quickly spread to China. This study aimed to investigate the disease's evolving epidemiological history, as well as analyze the clinical and CT imaging characteristics, treatment regimens, and patients' prognosis.

Methods: This was a retrospective study whose cases were 64 patients with a confirmed diagnosis of COVID-19. The clinical data were obtained from patients who were admitted to the isolation ward from 21 January 2020 to 19 February 2020.

Results: 60 out of 64 patients had a definitive history of exposure to people who had traveled from Wuhan City. The median time from onset of symptoms to first hospital admission was $3.9 \pm 1.9$ days. The initial symptoms included fever (46/64), dry cough (38/64), fatigue or myalgia (23/64), sore throat (10/64), diarrhea (3/64) along with late-onset symptoms like chest pains (2/64) and headaches (2/64). The majority of the patients (43/64) had normal white blood cell counts while $29.7 \%(19 / 64)$ had leukopenia. Only two patients $(3.1 \%)$ presented with leukocytosis. 58 of the 64 patients had abnormal radiological findings on chest CTs. The first chest CTs (within 2 days) was more sensitive in detecting COVID-19 infection (85.9\%) compared to the initial RT-PCR (56.3\%; $\mathrm{p}$ <0.01). The CTs showed lesions in multiple lung lobes in three-quarters of the patients while $15.6 \%$ had lesions localized to one lobe. Most of the lesions were typically dense with ground-glass opacity co-existing with consolidation or cord-like shadows. Most of these patients (50/64) have recovered and got discharged giving a mean length of hospital stay of $13.5 \pm 4.8$ days. Our hospital unit has not reported any COVID-19 related death so far.

Conclusions: Early intervention in COVID-19 disease improves patients' prognosis. Our data demonstrate the superiority of early radiological tests ahead of RT-PCR. The initial and dynamic CT changes in COVID-19 patients along with other clinical data shared above can better guide clinical decision making.

\section{Background}

In December 2019, Wuhan city in Hubei province of China, became the focus of the world owing to an outbreak of pneumonia-like Coronavirus disease 2019 (COVID-19) [1]. Epidemiological evidence suggested that the virus was likely of a zoonotic origin related to the city's seafood market [2]. However, the exact source of the new virus is not completely clear. The gene sequence of the virus from COVID-19 patients was highly homologous to that which exists in bats. In addition, this virus is also similar to the coronavirus which causes severe acute respiratory syndrome (SARS) [3-4]. The disease is highly contagious. As of 24 February 2020, the confirmed patients infected with COVID-19 were 79 331, including 2069 people spread across 29 other countries [5]. These figures are being updated daily and expected to increase further.

A previous study reported the clinical characteristics of the 137 COVID-19 patients admitted at the tertiary hospitals located in Wuhan city areas, contributing to an understanding of the epidemiological, clinical, laboratory, and radiological characteristics as well as treatment and clinical outcomes of those patients [6]. Another study found a familial cluster of SARS-Cov-2, clearly suggesting human to human transmission in family homes [7]. Furthermore, another study showed that as of 26 January 2020, the clinical characteristics of the 62 COVID-19 patients outside of Hubei province (wherein lies Wuhan city), had mild or less severe symptoms when compared to COVID-19 patients in Wuhan city [8]. Only one patient was reported discharged in this study [8]. During this time of the Chinese New Year break, many people (migrant workers and business owners alike) traveled out of the epicenter, Wuhan city, back to their home towns and abroad possibly spreading the disease to other places including Wenzhou city. During the course of treatments, we found that the patients' clinical manifestations are somewhat different from those being reported in Wuhan, and we also had discharged 50 patients. As a result, we herein describe the clinical manifestations, laboratory test results, imaging characteristics, and other findings among the COVID-19 patients in Wenzhou city as this may give more insight into the prevention and treatment options in this epidemic.

\section{Methods}

\section{Data sources and study population}


A retrospective research study was conducted to review the clinical and epidemiological characteristics of COVID-19 cases in Wenzhou city. Clinical data recorded from 21 January 2020 to 19 February 2020 were collected and analyzed. Since the onset of the COVID-19 outbreak, strict precautionary measures have been taken in Wenzhou city, Zhejiang province. For instance, fever clinics were opened to treat fever patients as well as to screen patients suspected to have COVID-19. The clinicians specifically focus on the patients with a history of travel to Wuhan or have had exposure to infected people within two weeks before the onset of illness since January 2020. Only patients with laboratory confirmation of infection with COVID-19 were eligible and enrolled in this study. All the 64 patients, whose data were used in this study, were admitted to the isolation ward of our teaching hospital. The data obtained included general health status, clinical manifestations or presentations, laboratory test results, CT scan results, and the treatment regimens they received.

Epidemiological information, such as date of onset of current illness and previous hospital admissions, was also collected. We also investigated the possibility of familial clusters, that is, index patients who traveled to Wuhan and infected their relatives and friends. The incubation period refers to the time from exposure to the onset of illness, which is estimated only among patients who could provide the exact date of close contact with people from Wuhan with confirmed or suspected COVID-19 infection. All of this data was collected by specifically trained clinicians who were utilizing standard reporting forms to ensure the accuracy and authenticity of the data.

\section{Laboratory Confirmation And Treatment}

After admission to our hospital, respiratory tract samples including sputum and nasopharyngeal swabs were collected from all the patients. These samples could be used to identify influenza, avian influenza, respiratory syncytial virus, adenovirus, parainfluenza virus, SARS-CoV, and MERS-CoV, as well as applicable to routine bacterial, fungal, and other pathogenic microorganism tests. Laboratory confirmation of COVID-19 was by real-time reverse transcription-polymerase chain reaction [9]. Other supplementary laboratory tests included complete blood counts, serum biochemistry, and C-reactive protein (CRP) assay. According to the current treatment guidelines [10], all patients received interferon-alpha (inhaled-50 $\mu \mathrm{g}$, twice daily) along with antiviral treatment lopinavir (400 mg, twice daily) and ritonavir (100 mg twice daily), and arbidol (200 mg, twice daily). The guideline does not recommend taking more than three antiviral drugs. Based on individual clinical needs, some of the patients also received Asmeton (73 mg, three times a day) and Cheno ( $0.3 \mathrm{~g}$, three times a day). In addition, patients whose resting respiratory rate was more than 30 breaths per minute, oxygen saturation was below $93 \%$, or multiple pulmonary lobes showed more than $50 \%$ progression of disease within 48 hours on chest CT received low dose corticosteroid treatment (40-80 mg per day) and gamma globulin (15-20 g per day) for 3-5 days [8, 10]. Release from medical isolation and discharge standards were as follows: 1. Temperature returns to normal for more than 3 days. 2. Significant improvement in respiratory symptoms. 3. Improved evidence on chest radiography. 4 . The results of two realtime reverse transcription-polymerase chain reaction tests taken 24 hours apart were negative for COVID-19 antigens. In addition to meeting the above four conditions, the patients who get discharged from hospital-based quarantine remain in intensive community based medical observation for 14 days after discharge [10].

\section{Statistical analysis}

We used the chi-square test to test the difference between the sensitivity of first chest CTs (early phase, $0-2$ days) for COVID-19 infection and the sensitivity of the initial RT-PCR. For continuous variables, we computed means and standard deviation or medians with interquartile ranges. For categorical variables, we computed the percentages of patients in each category. All analyses were performed using the SPSS software, version 22.0.

\section{Results}

\section{Epidemiological characteristics}

From January 21 to February 19, 2020, clinical and epidemiological data of 64 patients with confirmed COVID-19 infection were collected in Wenzhou. Out of 64 patients, 29 were male, 35 were female, aged $14-72$ years (mean age $46.1 \pm 13.1$ years), height

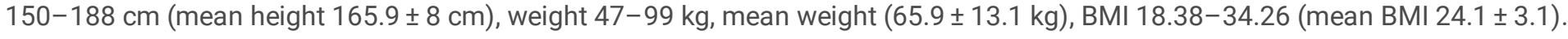
None of them had a history of exposure to the Wuhan seafood market. However, 60 out of 64 patients had a definitive history of 
exposure to individuals with confirmed COVID-19 infection. Among the 60 patients, 29 resided in Wuhan city, 3 had traveled to Wuhan within the last 14 days prior to admission while the remaining 28 patients had a positive history of exposure to individuals with confirmed COVID-19 infection. There were four patients who had no known history of exposure. Forty-seven patients provided the exact date of close contact with people who were confirmed or suspected to have COVID-19 infection. Twenty-nine patients (45.3\%) had a history of familial clusters. The incubation period was about 3 (1-9) days and the median time from onset of symptoms to first hospital admission was $3.9 \pm 1.9$ days (Table 1 ).

Table 1

Demographic and general characteristics of COVID-19-infected patients included in the study ( $\mathrm{N}$ $=64)$.

\begin{tabular}{|lll|}
\hline Items & $\mathbf{n}$ & Percentage, $\%$ \\
\hline Age-ranges (years) & & - \\
\hline$<30$ & 6 & 9.4 \\
\hline $30 \sim 40$ & 15 & 23.4 \\
\hline $40 \sim 50$ & 18 & 28.1 \\
\hline $50 \sim 60$ & 13 & 20.3 \\
\hline$>60$ & 12 & 18.8 \\
\hline Gender & & \\
\hline Male & 29 & 45.3 \\
\hline Female & 35 & 54.7 \\
\hline Height (SD)(cm) & $165.9(8)$ & - \\
\hline weight (SD)(Kg) & $65.9(13.1)$ & - \\
\hline BMI(SD) & $24.1(3.1)$ & - \\
\hline History of exposure to HuananSeafood & 0 & 0 \\
\hline Coexisting conditions & 14 & 21.9 \\
\hline Hypertension & 11 & 17.9 \\
\hline Diabetes & 5 & 7.8 \\
\hline liver disease & 2 & 3.1 \\
\hline Familial cluster & 29 & 45.3 \\
\hline The incubation period (days) & 3 & - \\
\hline Time from illness onset to first hospital admission (SD) (days) & $3.9(1.9)$ & - \\
\hline & & \\
\hline
\end{tabular}

\section{Clinical Features}

Fourteen of the 64 patients (21.9\%) had some pre-existing conditions, among them 11 (17.9\%) had hypertension, 5 patients (7.8\%) had diabetes while 2 patients (3.1\%) suffered from liver disease. 29 of the 64 patients (34\%) were related to familial clusters. The initial symptoms reported by patients included fever (46/64, 71.9\%), coughing (38/64, 59.4\%), fatigue or muscle pain $(23 / 64,35.9 \%)$, sore throat $(10 / 64,15.6 \%)$, diarrhea $(3 / 64,4.7 \%)$ along with other late-onset symptoms such as chest pain $(2 / 64,3.1 \%)$, and headaches $(2 / 64,3.1 \%)$ (Table 2). On admission, $67.2 \%$ (43/64) of the patients had normal white blood cell counts, 29.7\% (19/64) of the patients had decreased white blood cell counts and only $3.1 \%(2 / 64)$ had increased white blood cell counts. In addition, $71.9 \%$ $(46 / 64)$ had normal leukomonocyte counts. Nearly a third (17/64) had decreased leukomonocyte counts. Nearly three quarters (73.4\%, 47/64) of the patients had increased CRP (Table 3). 
Table 2

Clinical manifestations and physical signs of COVID-

19-infected patients in the study $(\mathrm{N}=64)$.

\begin{tabular}{|lll|}
\hline Items & $\mathbf{n}$ & Percentage, \% \\
\hline Initial symptoms & & \\
\hline Fever & 46 & 71.9 \\
\hline$<37.3^{\circ} \mathrm{C}$ & 18 & 28.1 \\
\hline $37.3^{\circ} \mathrm{C}-38.0^{\circ} \mathrm{C}$ & 25 & 39.1 \\
\hline $38.0^{\circ} \mathrm{C}-39.0^{\circ} \mathrm{C}$ & 16 & 25.0 \\
\hline$>39.0^{\circ} \mathrm{C}$ & 5 & 7.8 \\
\hline Cough & 38 & 59.4 \\
\hline fatigue or muscle pain & 23 & 35.9 \\
\hline pharyngalgia & 10 & 15.6 \\
\hline diarrhea & 3 & 4.7 \\
\hline chest pain & 2 & 3.1 \\
\hline headache renal dysfunction & 2 & 3.1 \\
\hline
\end{tabular}

Table 3

Laboratory test data for COVID-19-infected patients in the study $(\mathrm{N}$ $=64)$.

\begin{tabular}{|lll|}
\hline Items & $\mathbf{n}$ & Percentage, $\%$ \\
\hline White blood cell count, $\times 10^{9} / \mathrm{L}$ & & \\
\hline$<4$ & 19 & 29.7 \\
\hline $4-10$ & 43 & 67.2 \\
\hline$>10$ & 2 & 3.1 \\
\hline Lymphocyte count, $\times 10^{9} / \mathrm{L}$ & & \\
\hline$<1.0$ & 17 & 26.6 \\
\hline$>1.0$ & 47 & 73.4 \\
\hline Elevated C-reactive protein & 47 & 73.4 \\
\hline Elevated aminotransferase & 9 & 14.1 \\
\hline Abnormal myocardial enzyme profile & 3 & 4.7 \\
\hline RT PCR testing & & \\
\hline Initial RT-PCR Positive & 36 & 56.3 \\
\hline Any Positive RT-PCR & $64 / 64$ & 100 \\
\hline
\end{tabular}

The majority $(90.6 \%, 58 / 64)$ of the patients had abnormal chest CT findings. The sensitivity of first chest CTs (early phase, $0-$ 2 days) for COVID-19 infection was $85.9 \%$ (55/64) compared to the sensitivity of the initial RT-PCR was 56.3\% (36/64). This difference was statistically significant $(p<0.01)$. Interestingly, a 26 years old man came to the hospital because of diarrhea and fever $\left(38.1^{\circ} \mathrm{C}\right)$, his initial RT-PCR was positive, while on three occasions his chest CT scan results were negative (the times were January 23, January 27 and February 2), and he was discharged after an 11 day stay in the hospital. The remaining 20 patients had 
positive chest CTs while their initial RT-PCRs were negative. The chest CTs showed that $15.6 \%(10 / 64)$ of the patients had opacities only in one lobe per lung while $3.1 \%(2 / 64)$ had at least two lobes with opacities in each lung. Nearly three quarters $(71.9 \%, 46 / 64)$ of the patients had bilateral opacities and consolidation in their lungs (Table 4). A typical CT scan of COVID-19 patients showed bilateral or multiple lobular or subsegmental areas of dense, ground-glass opacity that co-existed with consolidation or cord-like shadows (Fig. 1). Repeated CT scans of COVID-19 patients can provide an important basis for ongoing clinical reasoning and decision making (Fig. 2).

Table 4

Chest CT imaging data for COVID-19-infected patients in the study $(\mathrm{N}=64)$.

\begin{tabular}{|l|ll|}
\hline Items & $\mathbf{n}$ & Percentage, \% \\
\hline Total Chest CT & 186 & \\
\hline The average number of Chest CTs per person & 2.9 & \\
\hline Ground-glass opacities and Consolidation & & \\
\hline Absence of Both Ground-Glass Opacities and Consolidation & 6 & 9.4 \\
\hline Presence of Either Ground-Glass Opacities or Consolidation & 58 & 90.6 \\
\hline Presence of Ground-Glass Opacities without Consolidation & 40 & 62.5 \\
\hline Presence of Ground-Glass Opacities with Consolidation & 15 & 23.4 \\
\hline Presence of Consolidation without Ground-Glass Opacities & 3 & 4.7 \\
\hline First Chest CT Positive & 55 & 85.9 \\
\hline Number of lobes affected & & \\
\hline 0 & 6 & 9.4 \\
\hline 1 & 10 & 15.6 \\
\hline$\geq 2$ lobes in one lateral lung & 2 & 3.1 \\
\hline$\geq 2$ lobes in bilateral Lungs & 46 & 71.9 \\
\hline
\end{tabular}

Although the epidemic lacks known effective drugs, our management followed the national guidelines provided for the treatment of the novel coronavirus pneumonia [10]. Following the systematic and comprehensive treatments outlined in the guideline, as of 19 February 2020, most of the patients (50/64) had fully recovered and were discharged, while the other 14 are still admitted in the hospital. The average length of hospital stay was $13.5 \pm 4.8$ days. Among the patients, 62 patients diagnosed with a mild form of COVID-19 while the remaining 2 developed severe COVID-19 pneumonia and were transferred to ICU for aggressive acute respiratory distress syndrome management. One of them has since improved and left the ICU. None of the patients in our care died of the disease during the study period (Table 5). 
Table 5

Treatment regimen and prognosis for COVID-19-infected

patients in the study $(\mathrm{N}=64)$.

\begin{tabular}{|lll|}
\hline Items & $\mathbf{n}$ & Percentage, \% \\
\hline Treatment & & \\
\hline Antiviral treatment & 64 & 100 \\
\hline Antibacterial treatment & 13 & 20.3 \\
\hline Systemic corticosteroid treatment & 5 & 7.8 \\
\hline Respiratory support & & \\
\hline Nasal cannula & 58 & 90.6 \\
\hline Non-invasive ventilation & 0 & 0 \\
\hline Prognosis & & \\
\hline Improved discharged & 50 & 78.1 \\
\hline Inpatient treatment & 14 & 21.9 \\
\hline Death & 0 & 0 \\
\hline
\end{tabular}

\section{Discussion}

Coronaviruses are a class of enveloped RNA viruses that live in many places, including humans, other mammals, and birds. To date, six coronaviruses have been found to infect humans [11]. There was a serious outbreak of SARS-CoV in China between 2002 and 2003 [12, 13]. MERS-CoV also led to a serious epidemic in the Middle East in 2012, and caused mass mortality [14]. Cross-species infection and occasional spillover events may lead to the recurrent emergence of new coronaviruses [15]. In December 2019, Wuhan city in Hubei province of China became an epicenter of the new coronavirus outbreak. As of 24 February 2020, the confirmed people infected with COVID-19 were 79 331, including 2069 people across 29 other countries [5]. These figures are being updated and increasing daily.

During the work of treating COVID-19 patients, we observed peculiar differences of our patients' clinical features from those reported in early cases in Wuhan city. We had 50 patients who had recovered and got discharged. According to our data, none of the infected patients at our teaching hospital had been exposed to the Wuhan city seafood wet market, instead, 29 patients had a history of familial cluster interactions which strongly suggested human to human transmission. At present, the primary source of infection appears to be from the COVID-19 patients as well as the asymptomatic ones. Respiratory and close contact transmission are the main modes of transmission. In this vein, aerosol propagation may pose a grave risk of transmission, especially to those exposed to high concentrations of aerosols for a relatively long period of time in a confined environment [10]. Therefore, containment procedures such as quarantine, avoiding close contacts with suspected or infected people as well as mass health education, avoiding large gatherings and taking precautionary measures against exposure to COVID-19 should continue to be implemented to prevent large scale spread.

Compared with the initial patients infected in Wuhan city [6], the symptoms of patients in Wenzhou city are relatively mild. Wenzhou patients' initial symptoms included fever, coughing, fatigue or muscle pain, pharyngalgia, diarrhea and rarely chest pain and headache. Atypical initial symptoms also deserve particular attention. For example, there were 3 patients with COVID-19 who presented with diarrhea as the initial symptom at disease onset in this study. Such observations are important because they point to clinicians being alert to the potential of misdiagnosis and differential diagnosis follow up. Among the Wenzhou patients, the median time from onset of symptoms to first hospital admission was relatively shorter. The news of the epidemic was now widely publicized officially by the Chinese government, as such these patients were very concerned about their health status and hence these early health-seeking behaviors. This could be the reason why the patients in the current study had relatively mild clinical symptoms. None 
of them was admitted to the hospital for breathing difficulties, a sign of severe disease progression. Early intervention in the treatment of COVID-19 pneumonia increases the likelihood of better prognosis among patients.

Viral nucleic acid detection using RT-PCR is the current standard. Non-contrast chest CT scans should also be considered for early diagnosis of this viral disease. Fang et al, reported that 50/51 (98\%) patients had evidence of abnormal CT findings compatible with viral pneumonia at baseline while one patient had a normal CT. Such an observation was also confirmed in the current study. Of the 50 patients with abnormal CT results in Fang and colleagues' study, $36(72 \%)$ patients had typical CT scan manifestations (e.g. peripheral, subpleural ground-glass opacities, often in the lower lobes). In addition, difference in the detection rate on the initial CT scan (50/51 [98\%, 95\% Cl 90-100\%]) was significantly greater than observed from the first RT-PCR (36/51 [71\%, 95\%Cl 56-83\%]) (p <.001) [16]. Chung et al, also reported that the chest CT scans of 3 out of 21 patients were negative for viral COVID-19 pneumonia at initial presentation [17]. On the other hand, Xie et al reported that 5 out of $167(3 \%)$ patients had negative RT-PCR results for COVID19 at initial presentation despite having chest CT scan findings typical of viral pneumonia [18]. In our data, the sensitivity of first chest CTs (early phase, $0-2$ days) was $85.9 \%$ (55/64), the sensitivity of the RT-PCR was $56.3 \%$ (36/64) demonstrating that early chest CT scanning was more sensitive than the initial RT-PCR $(p<.001)$. There are some possible reasons for this: 1$)$ the specificity and sensitivity of the detection technique itself are not good enough; 2 ) variation in the detection rate of kits from different manufacturers; 3) low patient viral load; or 4) improper clinical sampling [16]. We support the view that chest CT scans should be used as part of routine examination of COVID-19 Pneumonia at admission, especially when RT-PCR test results are negative.

The health statuses of patients with COVID-19 sometimes change very rapidly. This may be related to many factors such as treatment plan and patient's premorbid physique as well as preexisting chronic diseases such as hypertension and diabetes. In clinical practice, some patients do have recurrent symptoms and suddenly slide into acute respiratory distress syndrome or multiple organ failure even after a relatively stable period of time. This phenomenon is thought to be related to the "cytokine storm syndromes" in the patient's body [19]. Although there are currently no vaccines or any known special cure for this disease, the treatment regimens provided for in the guideline for treating the novel coronavirus pneumonia [10] were able to increase the number of recovering patients, prevent deaths and shorten hospital stay. A lot of pathological changes could be happening in the lesioned lungs of the patients. The temporal characteristics observed on the initial CT imaging as we all as on follow up scans COVID-19 patients is a rich data source of the novel disease's pathogenesis. In most of these discharged cases, the early stage of admission ( $0-5$ days) chest CT usually shows Ground-Glass Opacities (GGO) and Consolidation [20]. However, with the progression of the disease, the GGO lesions gradually decrease, and consolidation increases at first and then remains relatively stable for some days. If the patient's condition gets controlled and improves, at the last stage of the disease, chest CT scans often show different degrees of pulmonary fibrosis in the lungs. This trend could help clinicians to make correct judgments which are good for the resolution of the disease. The characteristics, including temporal CT changes among COVID-19 patients, can provide a lot of important information that feeds into clinical decision making.

In the absence of effective drugs against COVID-19, the diagnosis and treatment protocol for novel coronavirus pneumonia manual continues to inform practice and would also benefit from data such as generated in this study for its future revisions. Previously, the large-dose glucocorticoids used to treat SARS caused some serious adverse reactions [21] and also failed to effectively decrease the mortality rate associated with the disease [22]. In light of this, we treated the patients with lower dose (30-80 mg/day) and for a shorter time (3-5 days) using methylprednisolone to alleviate the pulmonary exudates and inhibit a possible cytokine storm syndromes. However, the impact of such an adaptation requires further studies.

\section{Limitations Of This Study}

Our study has several limitations. Firstly, only 64 patients were included and the study was motivated by the need to timeously communicate outbreak-related information since the disease is rapidly spreading in other countries outside of China. Secondly, the patients were only from Wenzhou city, Zhejiang province in China. Most of the patients had mild COVID-19 Pneumonia, only 2 patients were diagnosed with severe COVID-19 pneumonia, as such, some clinical features of severe COVID-19 pneumonia were not observed. Thirdly, at the time of study submission, long-term clinical follow-up of discharged patients was not yet available, therefore, we do not know the long-term effect of COVID-19 pneumonia on the pulmonary functions of discharged patients.

\section{Conclusion}

Page 8/11 
Early intervention in the treatment of COVID-19 pneumonia improves the prognosis of patients. The characteristics, including temporal CT changes in COVID-19 patients, can provide clinicians with a lot of important information to better guide clinical decision making. The present study provided further evidence that the sensitivity of early chest CTs is better than that of the initial RT-PCR. The majority of patients appear to recover within 2 weeks.

\section{Abbreviations}

COVID-19

Coronavirus disease 2019;

CT

Computerized tomography;

RT-PCR

Real-time polymerase chain reaction;

WHO

World Health Organization;

SARS

Severe acute respiratory syndrome;

MERS

the Middle East respiratory syndrome;

ICU

Intensive care unit;

$\mathrm{Cl}$

Confidence interval;

\section{Declarations}

\section{Ethics approval and consent to participate}

The study protocol was reviewed and approved by the local Ethics Committee at the Third Affiliated Hospital of Wenzhou Medical University, Ruian, Zhejiang Province, China, and conducted in conformity to the Helsinki Declaration. The data used in this study was anonymous before its use.

\section{Consent for publication}

Not applicable.

\section{Availability of data and materials}

All data generated or analyzed during this study are included in this published article and its supplementary information files (Additional file 1).

\section{Competing interests}

The authors declare that they have no competing interests.

\section{Funding}

No funding

\section{Authors' contributions}

Yong-chou Li and Li-ping Fu conceived the study, Yuan-tong Gao and Ke Zhou coordinated the study, Yong-chou Li, Min Luo collected the data, Peng-cheng Ma and Fa-Huan Song performed statistical analyses, Yong-chou Li, Li-ping Fu drafted the paper, which was reviewed by all authors. All authors read and approved the final manuscript. 
Not applicable

\section{References}

1. Li Q, Guan X, Wu P, et al. Early transmission dynamics in Wuhan, China, of novel coronavirus-infected pneumonia. N Engl J Med, 2020 Jan 29. Doi: 10.1056/NEJMoa2001316. [Epub ahead of print]

2. Lu H, Stratton CW, Tang YW. Outbreak of Pneumonia of Unknown Etiology in Wuhan China: the Mystery and the Miracle. J Med Virol 2020; published online 16 January. Doi: 10.1002/ jmv.25678.

3. Ji W, Wang W, Zhao X, Zai J, Li X. Homologous recombination within the spike glycoprotein of the newly identified coronavirus may boost cross-species transmission from snake to human. J Med Virol 2020; published online 22 January. Doi:10.1002/jmv.25682.

4. Lu R, Zhao X, Li J, et al. Genomic characterization and epidemiology of 2019 novel coronavirus: implications for virus origins and receptor binding. Lancet 2020; published online 30 January. Doi: 10.1016/ S0140-6736(20)30251-8.

5. "Situation Report -35." World Health Organization, World Health Organization, 24 Feb. 2020, https://www.who.int/docs/defaultsource/coronaviruse/situation-reports/20200224-sitrep-35-covid-19.pdf?sfvrsn=1ac4218d_2.

6. Liu K, Fang YY, Deng Y, et al. Clinical characteristics of novel coronavirus cases in tertiary hospitals in Hubei Province. Chin Med J (Engl).2020 Feb 7. Doi: 10.1097/CM9.0000000000000744. [Epub ahead of print]

7. Chan JF-W, Yuan S, Kok K-H, et al. A familial cluster of pneumonia associated with the 2019 novel coronavirus indicating person to person transmission: a study of a family cluster. 2020 Feb 15;395(10223):514-523. Doi: 10.1016/S0140-6736(20)30154-9. Epub 2020 Jan 24.

8. Xiao-Wei Xu, Xiao-Xin Wu, Xian-Gao Jiang, et al. Clinical findings in a group of patients infected with the 2019 novel coronavirus (SARS-Cov-2) outside of Wuhan, China: retrospective case series. BMJ. 2020 Feb 27;368:m792. Doi: 10.1136/bmj.m792.

9. Huang C, Wang Y, Li X, et al. Clinical features of patients infected with 2019 novel coronavirus in Wuhan, China. Lancet 2020; published online 24 January. Doi:10.1016/S0140-6736(20)30183-5.

10. Diagnosis and Treatment Protocol for Novel Coronavirus Pneumonia (5th interim edition). China NHCOTPSRO. Available from: http:

//www.nhc.gov.cn/jkj/s3577/202002/a5d6f7b8c48c451c87dba14889b30147/files/3514cb996ae24e2faf65953b4ecd0df4.pdf.

11. Zhou P, Fan H, Lan T, Yang XL, Shi WF, Zhang W, et al. Fatal swine acute diarrhoea syndrome caused by an HKU2-related coronavirus of bat origin. Nature 2018; 556:255-258. Doi: 1038/s41586-018-0010-9.

12. Su S, Wong G, Shi W, Liu J, Lai ACK, Zhou J, et al. Epidemiology, genetic recombination, and pathogenesis of coronaviruses. Trends Microbiol 2016; 24:490-502. Doi: 10.1016/j.tim.2016.03.003.

13. Normile D. Infectious diseases. Battling SARS on the frontlines. Science 2003; 300:714-715. Doi: 1126/science.300.5620.714.

14. Habib AMG, Ali MAE, Zouaoui BR, et al. Clinical outcomes among hospital patients with Middle East respiratory syndrome coronavirus(MERS-CoV) infection. BMC Infect Dis. 2019 Oct 22;19(1):870. Doi: 10.1186/s12879-019-4555-5.

15. Zaki AM, Van Boheemen S, Bestebroer TM, Osterhaus AD, Fouchier RA. Isolation of a novel coronavirus from a man with pneumonia in Saudi Arabia. N Engl J Med 2012; 367:1814-1820. Doi: 10.1056/NEJMoa1211721.

16. Yicheng Fang, Huangqi Zhang, Jicheng Xie, et al. Sensitivity of Chest CT for COVID-19: Comparison to RT-PCR. 2020 Feb 19:200432. Doi: 10.1148/radiol.2020200432.

17. Chung M, Bernheim A, Mei X, et al. CT Imaging Features of 2019 Novel Coronavirus (2019-nCoV). 2020 Feb 4:200230. Doi: 10.1148/radiol.2020200230. [Epub ahead of print]

18. Xie, X, Zhong, Z, Zhao, W, Zheng, C, Wang, F, Liu, J. Chest CT for typical 2019-nCoV pneumonia: relationship to negative RT-PCR testing. Radiology 2020. https://doi.org/10.1148/radiol.2020200343. Published online Feb 12, 2020.

19. GUPTA K K, KHAN M A, SINGH S K. Constitutive inflammatory cytokine storm: a major threat to human health. J Interferon Cytokine Res, 2020,40(1):19-23.

20. Pan Y, Guan H, Zhou S, et al. Initial CT findings and temporal changes in patients with the novel coronavirus pneumonia (2019nCoV): a study of 63 patients in Wuhan, China. Eur Radiol.2020 Feb 13. Doi: 10.1007/s00330-020-06731-x. [Epub ahead of 
print]

21. Griffith JF, Antonio GE, Kumta SM, Hui DS, Wong JK, Joynt GM, et al. Osteonecrosis of hip and knee in patients with severe acute respiratory syndrome treated with steroids. Radiology 2005; 235:168-175. Doi: 10.1148/radiol.2351040100.

22. Ho Guideline on management of severe acute respiratory syndrome (SARS). Lancet 2003; 361:1313-1315. Doi: 10.1016/s01406736(03)13085-1.

\section{Figures}

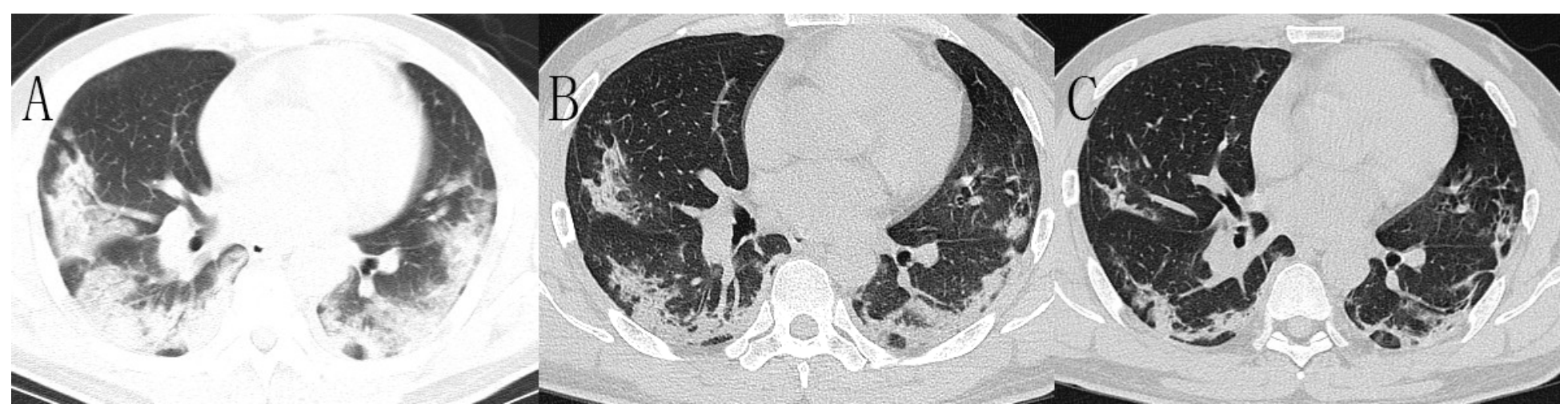

Figure 1

Large-scale pulmonary interstitial changes and fibrosis evolution in a patient with COVID-19. Male, 45-50 years old, fever for 6 days accompanied by chills, cough expectoration, with COVID-19 confirmed patients close contact history. A: CT examination on the day of admission, an axial CT image shows two lungs scattered multiple consolidative opacities, with a striking peripheral distribution in the two lower lobes. B 8 days later, an axial CT image shows the lesions were partially absorbed and the range was reduced. C: 17 days later, an axial CT image shows the range of lesions was further reduced, two lungs scattered multiple rough strips, which suggested pulmonary fibrosis.
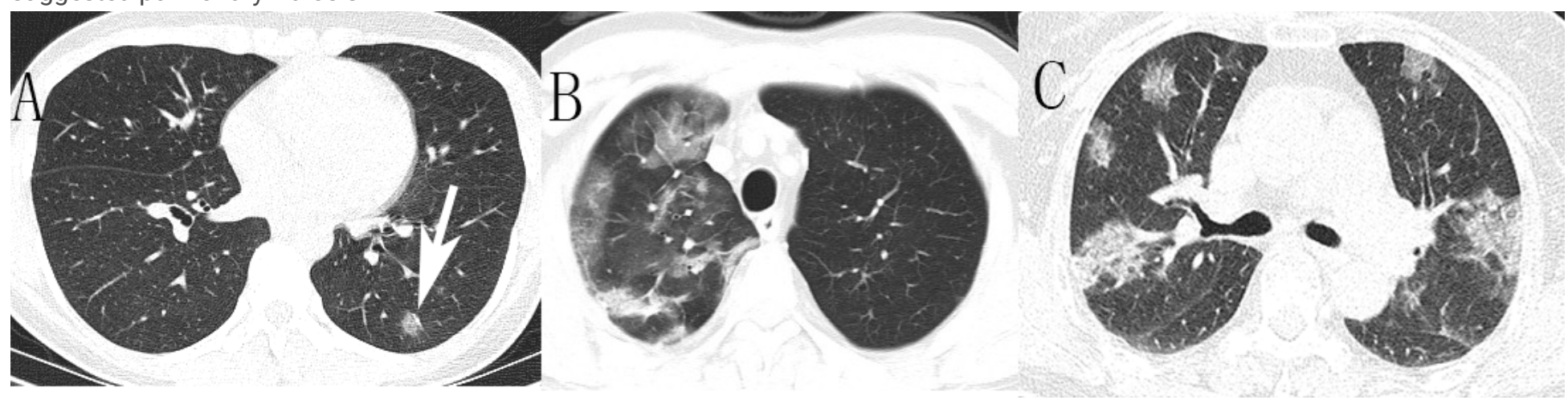

Figure 2

Typical lung manifestations in 3 patients with COVID-19. An axial CT image obtained without intravenous contrast in a 30-35 yearold female (Panel A) shows ground-glass opacities in the left lower lobe with a rounded morphology (arrow). An axial CT image obtained in a 60-65 year-old male (Panel B) shows larger ground-glass opacities in the right upper lobe. An axial CT image obtained in a 65-70 year-old female (Panel C) shows two lungs scattered multiple ground-glass opacities and consolidative opacities, with a striking peripheral distribution. 